\title{
Différentes techniques de réhabilitation ou de remplacement sans tranchée de conduites de distribution d'eau potable et d'assainissement
}

\author{
V. Ilotte $(*)$ J. Schroeyers $\left({ }^{* *}\right)$ G. Lehman $(* *)$ \\ (*) Compagnie Générale des Eaux, 52, rue d’Anjou 75008 Paris
}

(**) Sade, 28 rue de la Baume 75008 Paris

\section{Introduction}

Le Service Distribution de l'Exploitation de la Banlieue de Paris de la Compagnie Générale des Eaux (régisseur du service de l'eau du Syndicat des Eaux d'Ille-de-France), cherche de plus en plus à réduire les nuisances occasionnées par les travaux réalisés sur le réseau.

En étroite collaboration avec la Sade qui possède une importante expérience, non seulement dans le domaine de l'adduction d'eau potable, mais aussi dans celui de l'assainissement, de nouveaux procédés sont développés et testés, permettant ainsi d'être à l'avant-garde des techniques d'intervention sans tranchée.

Quelques-uns des procédés couramment utilisés pour l'adduction d'eau potable et pour l'assainissement, telles que les techniques de remplacement place pour place et de chemisage, sont présentés ici.

Remplacement "place pour place » des conduites de distribution d'eau

Le remplacement des conduites vétustes en zone urbaine se heurte souvent à des difficultés pour trouver un nouveau tracé satisfaisant, du fait :

— de l'encombrement du sous-sol dû aux différentes installations existantes ;

- des contraintes imposées par le gestionnaire de la voirie qui souhaite que les conduites soient installées sous trottoir.

Par ailleurs, l'abandon d'une conduite vétuste dans le sol est de plus en plus contesté par les gestionnaires du domaine public.

Ce contexte incite les gestionnaires de réseaux et les entreprises à développer des procédés qui permettent, soit l'utilisation de l'ancienne conduite, soit sa destruction pour en utiliser l'emplacement.
La Compagnie Générale des Eaux et la Sade, dans le cadre des travaux du Syndicat des Eaux d'Ille-de-France, mettent en œuvre deux procédés répondant à ces objectifs :

- l'extraction de conduites de 60 à $100 \mathrm{~mm}$ en fonte grise, pour les remplacer par du PEhd $\varnothing 125 \mathrm{~mm}$;

- le tubage adhérent, dit "Swage Lining ", de l'ancienne conduite par un tuyau PEhd.

La Sade également commercialise en France le procédé d'éclatement " Muscle ", surtout pour l'assainissement.

Extraction de conduites de $\varnothing 60$ à $100 \mathrm{~mm}$ en fonte grise

Le principe a déjà été développé dans des conférences précédentes, "NO DIG ", sous le nom de méthode berlinoise.

La conduite est extraite par tronçons, dont la longueur maximale est de 20 à $30 \mathrm{~m}$, par application d'une force de 30 à 50 tonnes transmise par des barres ou des câbles; au fur et à mesure de l'extraction, le tuyau en fonte grise est brisé et la nouvelle conduite est tractée simultanément (fig.l).

L'originalité du matériel développé par la Sade est d'utiliser comme moyen de traction un vérin de levage Freyssinet et les câbles de précontrainte associés. Le vérin est solidaire d'un bâti qui permet la reprise de l'effort de traction sur la paroi de la fouille ainsi que le débattement nécessaire à l'extraction du dernier élément de canalisation. Le vérin est animé d'un mouvement alternatif autorisant une course de $300 \mathrm{~mm}$.

Au fur et à mesure de l'extraction, les câbles sont conditionnés dans des enrouleurs (fig. 2 - 3).

Les anciens tuyaux en fonte grise sont éclatés par une pièce en forme de fer de lance prenant appui, soit sur un tronçon de canalisation non sollicité, soit sur le bâti du vérin Freyssinet. 

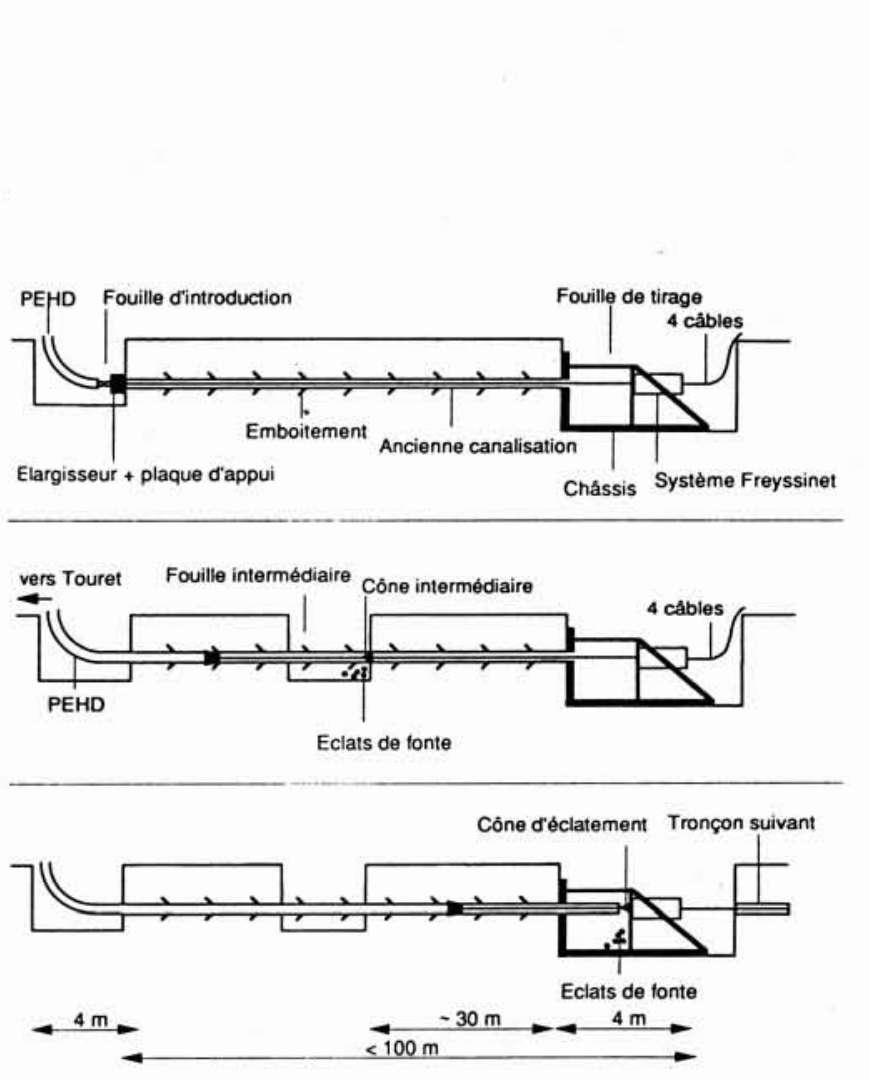

1.

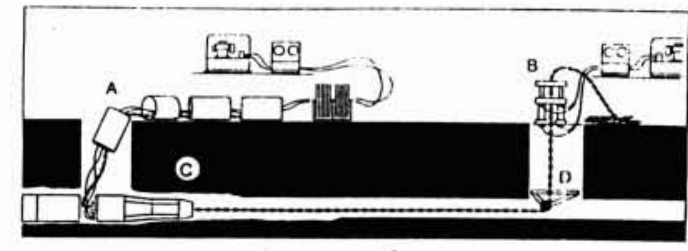

4

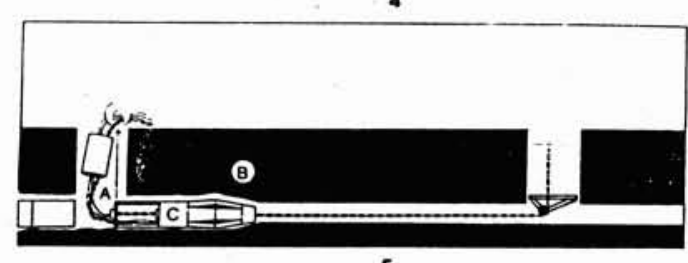

5
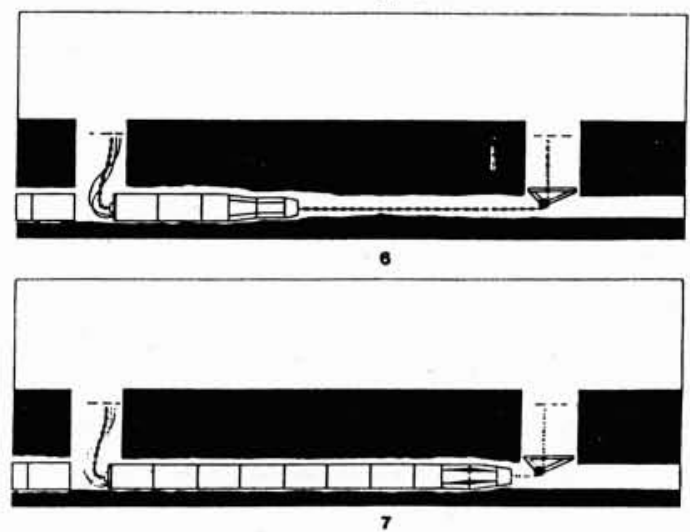

4 à 7. Résumé des différentes phases.

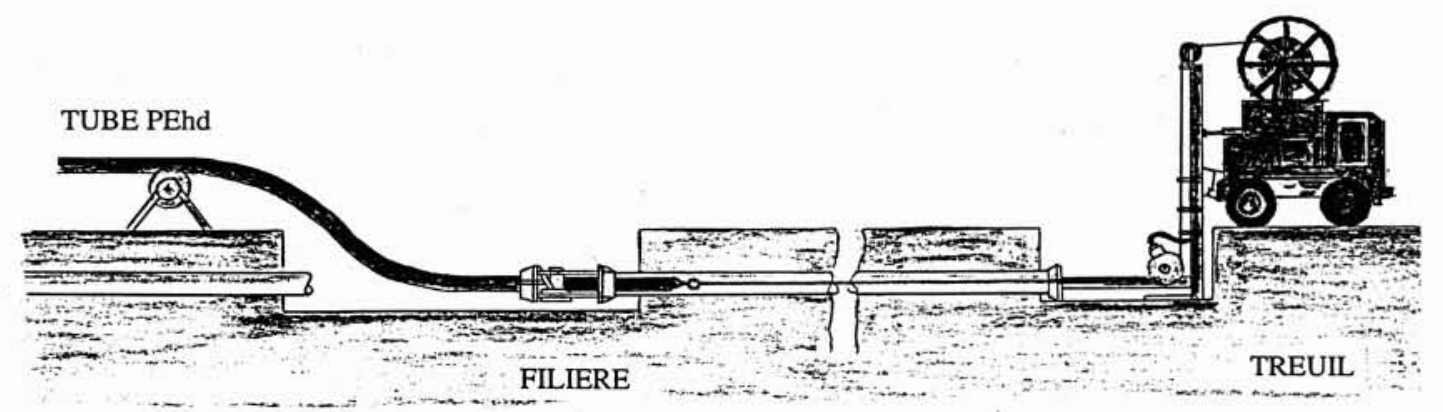

2. Tubage adhérent mis en auvre à température ambiante.

3. Matériel de tubage $(8,10$ et 12").

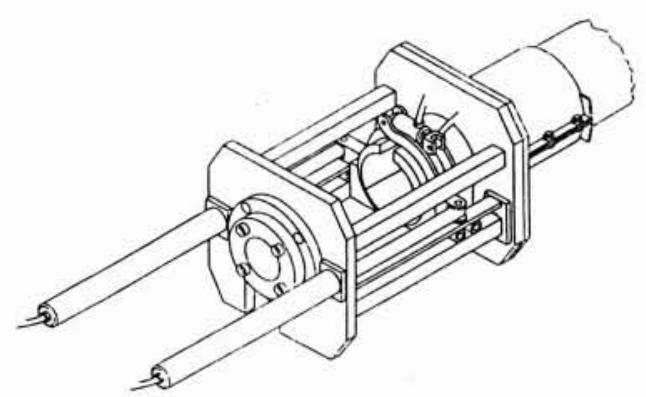


Par rapport au procédé d'éclatement, l'extraction permet de s'affranchir des sujétions liées à l'existence de pièces de réparation sur une canalisation vétuste, notamment des éléments en fonte ductile ou des manchons de réparation qui sont soit tronçonnés, soit démontés.

La présence de branchements est favorable à la mise en œuvre du procédé d'extraction, en ce sens qu'elle permet de bénéficier des fouilles de report des prises pour fractionner le bief à remplacer en tronçons d'une longueur maximale de $25 \mathrm{~m}$.

Comme toutes les techniques "place pour place», l'extraction nécessite la mise en place d'une conduite provisoire pour assurer la continuité de la distribution de l'eau.

Notre expérience porte actuellement sur l'extraction de conduites en fonte grise de $\varnothing 60,80$ et $100 \mathrm{~mm}$ remplacées par du PEhd $\varnothing 125 \mathrm{~mm}$ en couronne.

Sur des chantiers expérimentaux, l'économie sur les terrassements, les remblais et les réfections de sols, fait apparaître un coût identique à une pose en tranchée, malgré le surcoût dû à la mise en œuvre d'une conduite provisoire. L'amélioration du matériel et l'acquisition du savoir-faire par l'équipe devraient permettre de revoir les coûts à la baisse.

Enfin, il convient d'insister sur une caractéristique essentielle de ce procédé qui est le faible niveau sonore de l'intervention limité au seul bruit d'un groupe électrogène insonorisé de $50 \mathrm{kva}$.

\section{Le tubage adhérent "Swage Lining "}

Le "Swage Lining " est un procédé développé et breveté par British Gas. De nombreuses communications en ont déjà développé le principe que nous ferons que rappeler.

Le Swage Lining utilise les propriétés élastiques du polyéthylène. Le diamètre du tuyau en PEhd est réduit de 7 à $15 \%$ en le forçant par traction à travers une filière ; il est ainsi inséré dans la canalisation à rénover. Lorsque l'effort de traction est relâché, le tuyau tend à reprendre son diamètre initial, et vient alors se plaquer sur la paroi de l'ancienne canalisation.

Le tubage est ainsi exécuté, sans vide annulaire, avec une perte de section minimale (fig. 4 à 7 ).

Dans le cadre des travaux que la Compagnie Générale des Eaux et la Sade réalisent pour le Syndicat des Eaux d'Ille-de-France, les chantiers exécutés depuis la mi-91 en Swagelining concernent des canalisations vétustes en fonte grise de $\varnothing 200 \mathrm{~mm}$. Le tube PEhd utilisé est du PE $63 \varnothing$ $200 \mathrm{~mm}$ PN 10 pour des PMS ne dépassant pas 8 bars. Il reprend à lui seul la pression avec un coefficient de service d'au moins 1,63 à 50 ans. La mise en œuvre du tubage s'effectue à une vitesse constante comprise entre 1 et $2 \mathrm{~m} / \mathrm{min}$. Les divers raccordements et reports de branchements utilisent la technologie du polyéthylène.

Au cas où il est nécessaire de procéder à la coupe du tuyau PEhd, après sa mise en œuvre, pour insérer un té par exemple, il faut réaliser l'opération $24 \mathrm{~h}$ avant le raccordement, afin de permettre au tube PEhd tendu de retrouver un nouvel équilibre.
La propriété de relaxation du polyéthylène qui élimine dans le temps toutes les tensions résiduelles dues à la mise en œuvre, n'intervient de façon notable qu'à partir de 3 mois.

Par ailleurs, nous avons vérifié en laboratoire que les caractéristiques mécaniques et physiques du PEhd après rétraction n'avaient pas été altérées.

Nous avons constaté que malgré une diminution de section de $23 \%$, la capacité hydraulique de la canalisation avait été augmentée grâce à la faible perte de charge due aux tuyaux en PEhd.

$$
\begin{aligned}
& \text { Exemple : sur un tronçon de } 252 \mathrm{ml} \text { de fonte grise } \varnothing \\
& 200 \mathrm{~mm} \text { tubé en PEhd } \varnothing 200 \mathrm{PN} 10 \\
& \mathrm{~K}: \text { coefficient de rugosité de la formule de } \\
& \text { NIKURADSE } \\
& \text { pour } 100 \mathrm{~m}^{3} / \mathrm{h} \text { : avant } \mathrm{K}=3,30 \\
& \text { après } \mathrm{K}=0,08
\end{aligned}
$$

Sur le plan économique, les $1000 \mathrm{~m}$ réalisés à ce jour en $\varnothing 200 \mathrm{~mm}$ permettent d'escompter une économie de 10 à $20 \%$ par rapport au remplacement traditionnel, le gain étant fonction de la nature de la réfection de sol. Ceci devrait être confirmé dans la gamme des $\varnothing$ de 150 à $300 \mathrm{~mm}$.

En France, le procédé Swage Lining est mis en œuvre par la Sté TEMARE licenciée de British Gas; il est applicable $\mathrm{du} \varnothing 50 \mathrm{~mm}$ au $\varnothing 500 \mathrm{~mm}$ en mode structurant ou non.

\section{Le remplacement par éclatement " Muscle "}

Parmi les techniques de remplacement place pour place, il y a lieu de considérer le procédé d'éclatement " Muscle "; procédé breveté et développé pour l'Europe par la Sté CLEARLINE, et commercialisé en France par la Sade, qui en est le licencié exclusif.

Il consiste à briser l'ancienne conduite par un éclateur tiré par un treuil et un câble. Cet appareillage entraîne la nouvelle conduite en PEhd à sa suite.

L'éclateur est un outil en forme d'obus muni de pétales, qui s'écartent par un dispositif hydraulique, sans vibration, donc sans détérioration des ouvrages avoisinants et du terrain en place.

Les tuyaux existants doivent être " éclatables", comme le grès, l'amiante ciment, la fonte grise, le PVC et le béton non armé. Sont exclus les tuyaux tels que le béton armé, la fonte ductile et l'acier.

Pour l'eau potable, la nouvelle conduite est un PEhd continu introduit dans l'ouvrage par un puits d'accès spécialement aménagé.

Cette technique est valable également pour l'assainissement, où les accès se font uniquement par les regards, ce qui nécessite des tuyaux courts d'une longueur de $70 \mathrm{~cm}$, assemblés en fond de regard par un joint d'étanchéité et un "jonc" anti déboîtement. Ces tuyaux en PEhd sont, de plus, maintenus en compression par un casque indéformable situé à l'arrière et qui est plaqué par une chaîne intérieure et ceci pendant toute la durée de la mise en œuvre. 
Le nouvel ouvrage obtenu est parfaitement étanche.

Les phases successives de progression sont les suivantes :

— éclatement de la conduite par écartement des pétales ;

- tirage de l'éclateur par le treuil ;

- introduction d'un nouvel élément de tuyau (pour l'assainissement) dans le regard d'introduction.

Le diamètre de la nouvelle conduite par rapport à celui de l'ancienne, grâce au principe d'écartement, peut être maintenu et même augmenté. Il est possible par exemple de remplacer un $\varnothing 200 \mathrm{~mm}$ par un $\varnothing 250 \mathrm{~mm}$ ou même un $\varnothing 300 \mathrm{~mm}$.

Nous obtenons ainsi un nouvel ouvrage autostructurant, étanche et dont les caractéristiques hydrauliques sont celles bien connues du PEhd.

Dans le cas d'ouvrages profonds situés en ville et comportant de nombreux branchements d'assainissement, il est conseillé de raccorder ceux-ci à faible profondeur :

— soit en étoile jusqu'au regard le plus proche ;

- soit au moyen d'un collecteur parallèle se jetant dans le regard.

S'ils sont maintenus à leur emplacement initial, les branchements ne peuvent être repris que de l'extérieur, puisqu'il s'agit d'un procédé destructif.

Sur le plan économique, ce procédé permet d'atteindre des prix nettement inférieurs à ceux résultant d'une intervention traditionnelle, surtout dans les cas d'ouvrages nécessitant des blindages, l'évacuation des déblais, l'apport des remblais et la réfection de la chaussée. Par ailleurs, les gênes occasionnées au niveau des réseaux existants et de la vie urbaine sont considérablement réduites.

La gamme de diamètres, relevant de ce procédé, s'étend de $\varnothing 100 \mathrm{~mm}$ à $\varnothing 400 \mathrm{~mm}$.
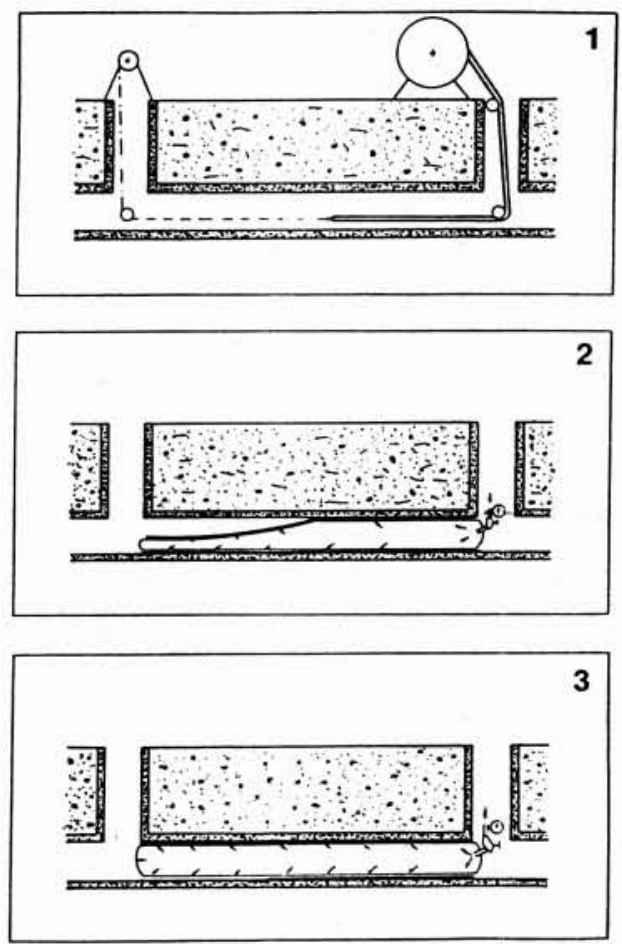

8. Schémas de mise en auvre.

\section{Chemisages}

Les deux procédés qui vont être présentés ont de nombreuses références pour les réseaux d'assainissement et d'eau potable.

Les procédés de chemisage Paltem et Copeflex sont mis en œuvre en France respectivement par Bonna et par la Sade.

Ces techniques s'inscrivent dans celles utilisées pour les opérations de réhabilitation. Les avantages en sont évidents d'un point de vue tant technique que social. Aucune tranchée ne doit être ouverte, à l'exception des deux fouilles aux extrémités de la canalisation.

Les procédés de chemisage consistent à insérer, dans une conduite existante, une nouvelle conduite en matériau plastique qui vient adhérer aux parois de l'ancienne. La perte de section, chemise en place, est négligeable ce qui conduit à un gain de débit assez important, grâce à l'amélioration du coefficient de rugosité, qui s'avère nettement inférieur pour le tuyau réhabilité.

\section{Le chemisage Copeflex}

Ce procédé de chemisage est un procédé français exploité par la Sade. Il consiste en la mise en place d'une chemise souple introduite par traction, puis gonflée et chauffee. Après durcissement par polymérisation, elle devient une nouvelle conduite intérieure autonome, sans aucun vide annulaire entre elle et l'ancienne (fig. 8).

Cette chemise est constituée de quatre éléments principaux assemblés en atelier :

- une membrane intérieure (en PVC pour l'assainissement, en PEhd pour l'eau potable) soudée en long, jouant, d'une part, le rôle d'étanchéité tel un ballon gonflable et, d'autre part, le rôle de protection anti-abrasive et chimique. Cette membrane est solidaire d'un feutre extérieur à enduire de résine époxy pour assurer la liaison PVC/armature ;

- une armature centrale constituée de strates tissés et non tissés de fibre de verre à épaisseur variable (selon calcul) et de résistances chauffantes intégrées parallèlement à l'axe de la canalisation;

- une enveloppe extérieure constituée par une membrane plastique, armée ou non, soudée en long à chaud, jouant le rôle de protection des éléments précédents au moment de la mise en œuvre ;

La mise en place, après les phases habituelles de préparation, consiste en :

- l'introduction de l'ensemble souple par un regard exis$\operatorname{tant}$;

- le tirage depuis le regard suivant ;

- la mise en pression d'air comprimé et le chauffage à

$70{ }^{\circ} \mathrm{C}$ environ;

- la polymérisation et le maintien en pression pendant la diminution de la température jusqu'à $40^{\circ} \mathrm{C}$ (généralement 3 à 4 heures);

- le découpage des extrémités. 
La durée d'un cycle complet de pose, pour un tronçon est d'une journée de travail. Les longueurs couramment réalisées sont comprises entre 50 et $120 \mathrm{~m}$.

Les avantages de ce procédé sont:

- l'étanchéité à $100 \%$ de la conduite rénovée ;

- la réalisation d'une conduite neuve auto-structurante (selon calcul) à l'intérieur de l'ancienne, considérée comme coffrage, avec reprise des charges intérieures et extérieures. Les caractéristiques ont été constatées par le CSTB, le Laboratoire de la ville de Paris, le CERCHAR.

Les branchements se font de l'intérieur pour les ouvrages visitables. Pour les ouvrages non visitables, ils sont réalisables de l'intérieur au moyen d'un robot caméra, mais l'étanchéité n'est pas garantie. Il est conseillé de les effectuer de l'extérieur pour assurer cette garantie.

Les diamètres traités actuellement sont compris entre $500 \mathrm{~mm}$ et $1600 \mathrm{~mm}$, mais il n'y a pas de limite supérieure.

\section{Le chemisage Paltem}

Le procédé Paltem a été développé et mis au point au Japon par Tokyo-Gaz et Ashimori, un fabricant de tuyaux de pompier (fig. 9).

Le processus de fabrication de tuyaux de pompier consiste à réaliser une gaine tissée qui, une fois enduite de résine côté extérieur, est retournée comme une chaussette avant d'être livrée. L'idée de base a donc été de mettre en œuvre le retournement d'une gaine tissée revêtue de résine (tuyau de pompier) à l'intérieur d'une conduite existante.
Le collage de l'une à l'autre est assuré par la résine mise en place à l'intérieur de la gaine avant l'introduction.

Ce procédé a maintenant plus de 20 années de référence sur le réseau de gaz au Japon.

La particularité des gaines homologuées par le Service Distribution de la Banlieue de Paris réside dans l'utilisation d'une gaine renforcée d'une spire en Kevlar permettant ainsi de reprendre les efforts de pression, indépendamment de la canalisation ancienne. Cette gaine particulière a fait l'objet d'une série de tests de résistance réalisés par le C.E.B.T.P. qui nous ont donné entière satisfaction pour ce qui concerne ses caractéristiques mécaniques.

\section{Conclusion}

Dans ce texte, nous avons présenté quelques uns des procédés de réhabilitation ou de remplacement sans tranchée. Cette liste ne prétend pas être exhaustive, mais permet d'avoir un aperçu des deux familles de techniques auxquelles on a le plus couramment recours.

Il est bien évident qu'aucune de ces techniques n'est optimale pour toutes les configurations possibles, dans lesquelles nous sommes amenés à travailler. Chacune a ses avantages et ses limites qui la rendent unique pour une application particulière donnée.

Voilà pourquoi nous avons, dans l'éventail des techniques à notre disposition, un si large nombre de procédés qui peuvent sembler être, au premier abord, en concurrence.

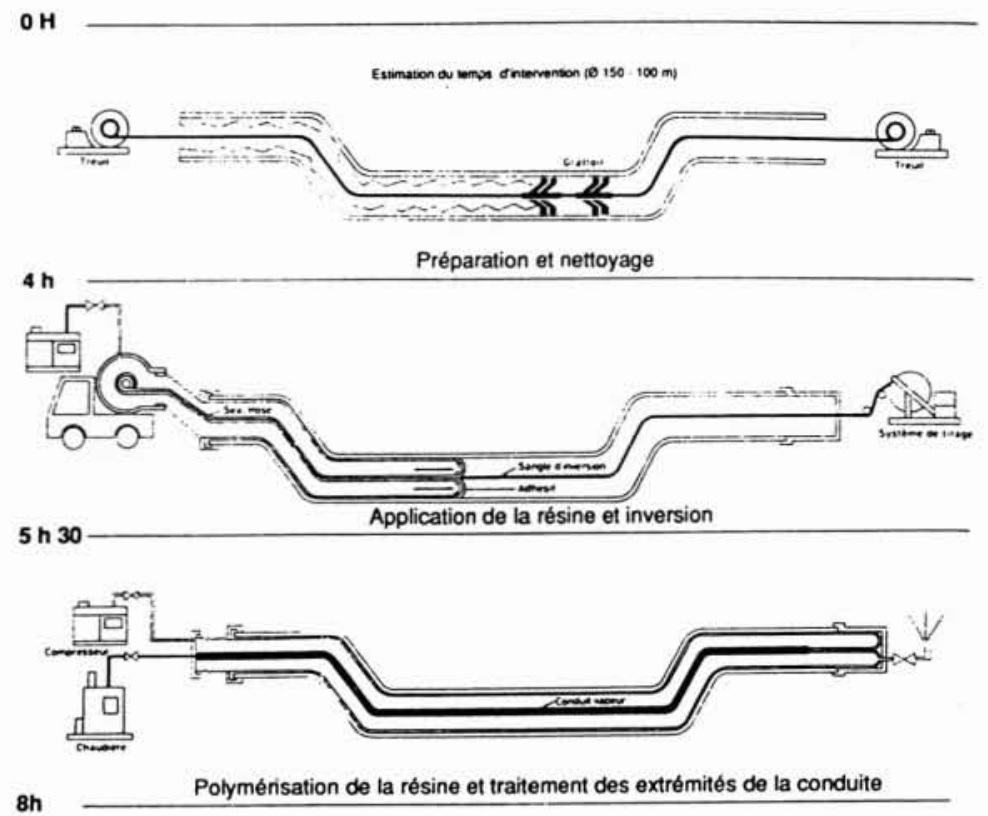

9. 